\title{
DEL TRÁNSITO INTERRUMPIDO A LA DEPORTACIÓN. NiÑAS, NIÑOS Y ADOLESCENTES CENTROAMERICANOS VIAJANDO SOLOS HACIA ESTADOS UNIDOS
}

\section{From AN INTERRUPTION IN TrANSIT TO DEPORTATION. Central American Girls, Boys and Adolescents Traveling Alone toward the United States}

\section{Carolina Rivera Farfán}

Resumen: El trabajo expone la problemática que involucra la migración de niñas, niños y adolescentes, entre 12 y 17 años, que proceden de países del Triángulo Norte de Centroamérica — Guatemala, Honduras y El Salvador- y viajan sin compañía hacia Estados Unidos. Ubica circunstancias puntuales que ayudan a entender la complejidad de una realidad que involucra a Centroamérica-México-Estados Unidos y que tomó relevancia mediática en junio de 2014 cuando el gobierno estadounidense hizo pública la noticia de la detención de más de cincuenta mil de ellos, migrantes no acompañados que ingresaron a ese país en calidad de indocumentado, quienes cruzaron más de una frontera internacionalmente reconocida sin sus padres o tutores. Migraron, y lo siguen haciendo, en separación o "sin compañía adulta" a quien la ley o las costumbres asignan esa responsabilidad. Para ello se hizo una revisión de textos e informes vinculados con la temática, así como la sistematización de información empírica construida a través

\footnotetext{
* Antropóloga del Centro de Investigaciones y Estudios Superiores en Antropología Social, México. Temas de interés: migraciones laborales y legislación, derechos humanos, niñez migrante, migración en América Central y sureste de México, pluralidad religiosa, https:// orcid.org/0000-0002-7596-722X, correo e.: crivera@ciesas.edu.mx

Fecha de recepción: 2808 17; Fecha de aceptación: 280618.
}

(cc) EY-No-ND Páginas 9-36. 
del ejercicio etnográfico realizado durante los años 2012 y 2013 en la ciudad fronteriza de Tapachula, Chiapas.

Palabras clave: niñez y adolescencia migrante; migración en tránsito; deportación; riesgo y vulnerabilidad en flujos migratorios; derechos humanos y vulnerabilidad.

Abstract: This paper discusses problems involving unaccompanied girls, boys and adolescents who emigrate from countries in the Northern Triangle of Central America - Guatemala, Honduras and El Salvador - to the United States. Without attempting to characterize the different particularities that this phenomenon produces, this exercise describes some specific situations that help to understand the complexity of a process that involves Central America-MexicoUnited States, and that became relevant to the mass media in June 2014 when the US government publicly announced the arrest of more than fifty thousand young unaccompanied migrants who entered its country as undocumented migrants. They are unaccompanied girls, boy and adolescents, aged 12 to 17 —although some are younger-, who cross an internationally recognized border, without their parents or guardians. They migrate in separation or without the company of the adults who, by law or by custom, are responsible for them. For this purpose, a review of texts and reports related to the subject was made, as well as the systematization of empirical information constructed through the ethnographic exercise carried out during the years 2012 and 2013 in the border city of Tapachula, Chiapas.

Keywords: Migrant children and adolescents; Migration in transit; Deportation; Risk and vulnerability in migratory flows; Human rights and vulnerability. 


\section{Introducción}

Este artículo procura un acercamiento a las circunstancias que viven niños, nińas y adolescentes centroamericanos de Guatemala, Honduras y El Salvador — países del Triángulo Norte de Centroamérica-, que ingresan por las diversas rutas que conectan la frontera de Guatemala y Chiapas, en el sur de México, y que tienen la intención de viajar hacia Estados Unidos. Por su condición de migrantes irregulares la mayoría son detenidos por autoridades migratorias mexicanas y posteriormente deportados, mediante la gestión consular de esos países en sus oficinas ubicadas en Tapachula, Chiapas. De forma similar son detenidos en Estados Unidos aquellos que consiguieron cruzar el territorio mexicano e internarse en los estados del sur de ese país. La investigación, en proceso, y la documentación citada nos indican que el problema presenta diversos desafíos y que su tratamiento requiere el compromiso y acción de los gobiernos de la región —Centroamérica, México, Estados Unidos- que vaya más allá de las políticas restrictivas de contención y expulsión, y posicionar el Interés Superior del niño, la niña y el adolescente en el primer plano.

El trabajo se refiere a personas que tienen entre 12 y 17 años, que cruzan una frontera internacionalmente reconocida, sin sus padres $o$ tutores. Migran en separación o "sin compañía adulta” a quien la ley o las costumbres asignen esa responsabilidad. ${ }^{1}$ Su detención y deportación en la actualidad se vincula con las estrictas políticas migratorias basadas en criterios de securitización y desestímulo del desplazamiento irregular de sur a norte. Estados Unidos ha instituido políticas migratorias altamente restrictivas y el gobierno de México —Programa Integral de la Frontera Sur- es su imprescindible socio y colaborador para llevar a cabo la contención, detención y deportación realizadas en condiciones que violan sus derechos en virtud de las leyes federales y el derecho internacional humanitario. Esta compleja realidad llegó a un punto álgido en junio de 2014 cuando autoridades migratorias de Estados Unidos reconocieron la detención de 56 mil niños y adolescentes migrantes irregulares procedentes de Guatemala, Honduras, El Salvador y México. La Casa Blanca declaró una "situación humanitaria

\footnotetext{
${ }^{1}$ Acnur, OIT, OIM, Unicef (2013).
} 
urgente". Sin embargo, la noticia en realidad no era tal: la migración de niños viajando solos se ha registrado desde mediados del siglo pasado, la novedad es el incremento del flujo de sur a norte. Entre 2009 y 2016 fueron detenidos 225,351 menores migrantes no acompañados en la frontera suroeste de Estados Unidos; de ellos 54.41\% provenía de esos tres países centroamericanos; $44.14 \%$ de México, y $1.45 \%$ de otra nacionalidad. ${ }^{2}$

Para su exposición el artículo se ha organizado de la manera siguiente: en la primera parte se hace una breve presentación de estudios sobre niños y niñas en los actuales procesos migratorios. La segunda, refiere a niñez y adolescencia que viaja sin compañía hacia Estados Unidos desde Centroamérica y México. El tercer apartado informa sobre el perfil migrante a partir de la pregunta ¿quiénes son y por qué emigran? Posteriormente, se abordan las síntesis de narrativas de algunos de ellos que fueron detenidos y resguardados en el albergue temporal del Sistema Nacional para el Desarrollo Integral de la Familia, DIF, en la ciudad fronteriza de Tapachula, Chiapas, en tanto se concretaba su proceso de devolución a sus países de origen. Los relatos pertenecen a un grupo de quince nińas, niños y adolescentes, retomados durante breves temporadas de campo que realicé en la ciudad de Tapachula durante los años 2012 y 2013. A partir del dato etnográfico y de información bibliográfica se explica el contexto y el marco en que estos actores experimentan situaciones de riesgo, incertidumbre e inseguridad en un proceso migratorio anulado al ser deportados. Después, el artículo se acerca a la identificación del contexto del problema y sus efectos, para finalizar con algunas reflexiones. La observación se sumó a las entrevistas, abiertas y estructuradas, a los principales actores involucrados: niñas, niños y adolescentes en tránsito por México; representantes de instancias gubernamentales y no gubernamentales; autoridades migratorias; cónsules de Guatemala y el Salvador; funcionarias de dos albergues del DIF y del Centro de Derechos Humanos Fray Matías de Córdova.

\footnotetext{
${ }^{2}$ Conapo et al. (2016).
} 


\section{Los niños en procesos migratorios}

Migrar es un fenómeno presente en la historia humana y los motivos de la movilidad son diversos, también las rutas y los caminos que llevan a la persona al lugar deseado, e incluso al destino no pensado o no planeado. Por razones económicas, por deseo o aspiración, por gusto, por reconocer nuevos horizontes o reunificarse con su familia, por un desplazamiento forzado - como huir de la violencia o por un desastre natural, entre los más comunes-, hoy día la migración o las migraciones de las personas forman parte de la vida cotidiana. Los niños, las niñas y los adolescentes no son ajenos a ellas y participan, de diversas maneras, en los procesos migratorios. Su "viaje", su desplazamiento, se ha ligado usualmente al de los adultos, ya sean sus padres, o uno de ellos, o cualquier otro adulto a quien la ley o las costumbres asignen esa responsabilidad. Sin embargo, también lo hacen solos, o en compañía de amigos, de vecinos, de algún pariente, o de los traficantes de personas -polleros, coyotes.

Si bien las razones y factores que impulsan la migración de estos actores son múltiples, aunque lo económico predomina, o es lo más visible en un inicio, es cierto que hay otras razones. En este caso, destaca la necesidad de la reunificación familiar, pero también sobresale el contexto de violencia — familiar, de género, comunitaria, social—, criminalidad e inseguridad ciudadana. Los últimos reportes indican que la violencia prevaleciente en los países del Triángulo Norte centroamericano ha desplazado la motivación económica, derivada de la desigualdad social y la precariedad económica, como principal causa para escapar de esa realidad. En un reciente informe de Médicos Sin Fronteras (2017) se puede leer que 39.2\% de migrantes en tránsito, entrevistados por integrantes de la organización, mencionó que la razón principal de la huida fue el haber sufrido ellos mismos o sus familias ataques directos, amenazas, extorsión, o haber sido objeto del reclutamiento forzoso por parte de bandas criminales. En este contexto, 43.5\% había perdido a algún familiar en un incidente violento en los dos años anteriores a la huida. En el caso de los salvadoreños, el porcentaje se situaba en 56.2\%, de ellos había sufrido chantaje o extorsión 54.8\%, porcentaje más alto que el de los encuestados originarios de Honduras 
o Guatemala (Médicos Sin Fronteras, 2017). Es decir, la violencia en esos países se ha posicionado en tal magnitud que resulta la principal causa de emigración, aunque la búsqueda y aspiración de mejoras económicas que apuestan encontrar en Estados Unidos está en el plano más visible.

La reunificación familiar es otra significativa motivación, particularmente de aquellos que fueron encargados a un pariente por varios ańos en tanto sus padres, o uno de ellos, emigraron. Pero también, entre los adolescentes de entre 12 y 17 años, la búsqueda personal, distante del control doméstico en el ámbito familiar, se vincula con las aspiraciones de iniciar procesos de relativa independencia. Blanco (2014) destaca otros factores que atraen a mujeres, jóvenes guatemaltecas, provenientes de zonas rurales, por ejemplo migran al sureste mexicano para insertarse en el trabajo doméstico. Es un aliciente poder salir de la casa paterna con el deseo explícito de "conocer otros lugares", y la ciudad para ellas es "símbolo de confort, de una calidad de vida superior" (Arriola, 1995: 118). Esto, en parte, motivado por lo que otras personas cercanas les han contado de Tapachula y de otros destinos. El imaginario de las ciudades, distante de la casa paterna, cautiva e impulsa de igual manera la migración entre las mujeres jóvenes y adolescentes.

Desde otro ángulo, Pavez (2012) ha estudiado la migración de niñas y nińos peruanos que migran hacia Santiago de Chile y Barcelona en el marco de un proyecto familiar migratorio. A partir de su pregunta “¿Quién decide la migración infantil?", la autora muestra que la participación de infantes es compleja, contradictoria, y presenta diferentes matices en cada situación debido a que los procesos migratorios no son lineales, en muchos casos comienza con la migración adulta y "termina" con la llegada de hijas e hijos. Sin embargo, no siempre ocurre así, por lo que llama a observar los arreglos y las estructuras familiares diversas en cada caso. Citando a Pedone (2010), Pavez coincide en que los procesos migratorios familiares siguen tendencias transnacionales complejas, particularmente en momentos de crisis, lo que genera movilidades familiares e infantiles de retorno y circularidad constantes e innovadoras (ídem: 106). Envuelta en esas complejidades la autora señala que no siempre los niños quieren, y no deciden viajar 
con sus padres, pues dejan toda una trayectoria de vida en su lugar de origen. En cualquier caso, los niños se ven afectados inevitablemente por la migración familiar, la cual cambiará sus vidas.

Justamente por la amplitud y diversidad de perspectivas del problema y sus causas, por ahora este artículo se concentra en la migración de aquellos que se desplazan "sin compañía" o "sin compañía adulta", hacia Estados Unidos por causas de reunificación familiar y por la amenaza de la violencia en sus diversas expresiones. Refiere a nińas, niños o adolescentes que migran en separación de su padre y madre - $\mathrm{O}$ tutor- y cuyos cuidados no los efectúa la persona adulta reconocida social y culturalmente como responsable de sus atenciones.

\section{Migración de niños, niñas y adolescentes centroamericanos que viajan sin compañía hacia Estados Unidos}

En el mes de junio de 2014, autoridades migratorias de Estados Unidos, mostrándose sorprendidas, reconocieron que habían ingresado a su país más de cincuenta mil niños y niñas migrantes irregulares procedentes Guatemala, Honduras, El Salvador y México. Tras su detención, el presidente Obama declaró una "situación humanitaria urgente”. La noticia en realidad no era tal; eso sí, la migración de niños centroamericanos ha registrado un notorio aumento. Se trata de una realidad que se ha venido presentando y registrando desde años anteriores no sólo en Estados Unidos sino también en México.

Durante los últimos dos años el Instituto Nacional de Migración, INM, de México incrementó la detención y devolución de niños y adolescentes provenientes de los tres países de Centroamérica. En 2015 la Comisión Nacional de Derechos Humanos, CNDH, tuvo registro de 20,368 nińos no acompañados detenidos por INM; en el transcurso de 2016, el Instituto detuvo a 40,114 menores de edad, de los cuales 2,176 eran no acompañados. En ese mismo año las cifras indicaron la detención de 23,096 personas, de las cuales 10,943 fueron niñas, niños y adolescentes viajando sin compañía adulta (CNDH, 2016).

Resulta complicado cuantificar o estimar metodológicamente los movimientos de estos sujetos debido a la amplia gama de variables que han de considerarse. Origen-destino, la repetición de los movimientos, 
eventos vs. personas, tiempos de estancia en los espacios de referencia y sobre todo la multiplicidad de tipos de migraciones (Corona, 2008). Cuantificar y caracterizar esta modalidad la reviste de una vasta complejidad. Las certezas que se tienen es que son niños, niñas $\mathrm{y}$ adolescentes que se desplazan por diversos rumbos, que han cruzado más de una frontera nacional y que enfrentan una situación de mayor riesgo, ya que les puede colocar en situaciones de vulnerabilidad. Ésta se concibe, en principio, por la condición de irregularidad migratoria, lo que les expone a situaciones de inseguridad que atentan contra sus derechos y facilita acciones como las de explotación sexual y laboral, trata y extorsión. Dicha situación se les plantea desde el momento mismo de su partida y se asocia a su condición indocumentada, así como con el plan elegido para emprender la travesía por los distintos países de tránsito como en el de destino — condición del viaje, cómo y quién o quiénes intervendrán.

No hay una cifra precisa sobre cuántos son los que ingresan irregularmente a México — por los puntos fronterizos entre Guatemala, Chiapas y Tabasco - ni a Estados Unidos. Es posible identificar las cantidades de los que son "asegurados" en las más de cincuenta garitas migratorias de México y que posteriormente se les detiene y conduce a la Estación Migratoria Siglo XXI, en Tapachula, para ser repatriados por autoridades mexicanas a sus países de procedencia. Entre los años 2009 y 2015 México regresó a 64,664 niños y adolescentes de los países del Triángulo Norte de Centroamérica.

Figura 1. Eventos de retorno asistido de menores centroamericanos por autoridades mexicanas: 2009-2015.

\begin{tabular}{|c|r|r|r|r|r|r|r|c|}
\hline \multicolumn{1}{|c|}{ País } & $\mathbf{2 0 0 9}$ & $\mathbf{2 0 1 0}$ & $\mathbf{2 0 1 1}$ & $\mathbf{2 0 1 2}$ & $\mathbf{2 0 1 3}$ & $\mathbf{2 0 1 4}$ & $\mathbf{2 0 1 5 ^ { * }}$ & $\begin{array}{c}\text { Total } \\
\text { país }\end{array}$ \\
\hline Honduras & 1,385 & 1,692 & 1,288 & 2,169 & 3,686 & 7,967 & 6,010 & 24,197 \\
\hline Guatemala & 2,073 & 1,933 & 1,935 & 2,393 & 3,012 & 6,196 & 10,104 & 27,646 \\
\hline El Salvador & 527 & 972 & 770 & 1,280 & 1,703 & 3,758 & 3,811 & 12,821 \\
\hline Total & 3,985 & 4,597 & 3,993 & 5,842 & 8,401 & 17,921 & 19,925 & $\mathbf{6 4 , 6 6 4}$ \\
\hline
\end{tabular}

${ }^{*}$ La información de 2015 hace referencia al periodo enero-septiembre.

Fuente: Conapo et al. (2016) 
Según esta fuente, la mayoría son hombres; sin embargo, a partir de 2013 los "eventos de retorno asistido" —así nombra INM la detención y deportación - de menores centroamericanos de ambos sexos aumentaron considerablemente, y Guatemala destaca como el país con más registros de devoluciones. En años recientes, resalta el incremento de menores retornados de sexo femenino (Conapo et al., 2016). Es una cifra sin precedentes que revela la consonancia que el Programa Frontera Sur tiene con los intereses del gobierno norteamericano al detener y deportar la migración irregular desde el sur. Sin embargo, no sólo centroamericanos son parte de las estadísticas de detención y deportación gestionadas desde México. Similar "suerte" afronta la niñez mexicana que migra hacia Estados Unidos ya sea por reunificación familiar y/o por las precarias condiciones estructurales en que viven millones de ellos; en términos absolutos, niños mexicanos representan la mayor cifra de deportación en la región, como se observa en la figura 2.

Figura 2. Menores migrantes centroamericanos y mexicanos no acompañados aprehendidos en la frontera suroeste de Estados Unidos 2009-2015*.

\begin{tabular}{|l|c|c|}
\hline \multicolumn{1}{|c|}{ País } & Absoluto & Relativo (\%) \\
\hline El Salvador & 39,522 & 17.54 \\
\hline Guatemala & 46,746 & 20.74 \\
\hline Honduras & 36,356 & 16.13 \\
\hline México & 99,466 & 44.14 \\
\hline Otros & 3,261 & 1.44 \\
\hline & $\mathbf{2 2 5 , 3 5 1}$ & $\mathbf{1 0 0 . 0 0}$ \\
\hline
\end{tabular}

*La información de 2015 hace referencia al periodo enero-septiembre. Fuente: Conapo et al. (2016)

Un informe del Consejo Nacional de Evaluación de la Política de Desarrollo Social (Coneval, 2015) indica que para 2014 un poco más de 40 millones de la población menor de 18 años en México vive con niveles de pobreza y vulnerabilidad. A esa realidad se agregan otros elementos, como son los patrones migratorios de familiares y amigos. Por más de cien años determinadas regiones del país se han caracterizado por haber sido expulsoras de migrantes hacia Estados Unidos. Algunas 
causas se vinculan con la desintegración o separación de familias migrantes que desean reunirse, así como la influencia económica y cultural de Estados Unidos, entre otras (IIDH, 2016).

\section{¿Quiénes son y por qué emigran?}

Son niñas, niños y adolescentes de entre 12 y 17 años, aunque hay menores, que cruzan de manera irregular una o más de una frontera internacionalmente reconocida, sin sus padres o tutores. Este sector de la población hasta muy recientemente empezó a ser considerado en los estudios de la migración interna e internacional debido a su presencia cada vez más numerosa. Para la frontera sur de México destacan las investigaciones de las migraciones laborales internas donde guatemaltecos, menores de 18 años, se han incorporado al sector agrícola y a otras ramas de la economía informal (Rojas, 2002, 2005; Rivera, 2014). Los estudios muestran, así como mis propias indagaciones, que las lógicas económicas son las principales motivaciones, pero también existen otros estímulos sociales y culturales que los empuja a desplazarse fuera de la tutela doméstica.

La Organización Internacional para las Migraciones -OIM, sede Tapachula, Chiapas - y la Organización Panamericana para la Salud, OPS, identificaron, a través de un estudio exploratorio en 2014, el acceso/oferta a los servicios de salud, particularmente el de la prevención, cuidados en el embarazo temprano e infecciones de transmisión sexual de niños, niñas y adolescentes centroamericanos en tránsito, así como de aquellos que prolongan o definen su estancia en la ciudad de Tapachula, sobre todo de jóvenes trabajadoras domésticas y de niñas y niños que trabajan sobre la calle en el comercio ambulante.

En el punto de las migraciones en la región centroamericana sobresalen los trabajos de Cranshaw y Morales (1998), Caballeros (2006), así como el de la Red Internacional de Migración y Desarrollo (RIMD, 2004) y Escobar Sarti (2008), los cuales identifican a los nińos y adolescentes como emergentes actores formadores de tendencias y como "nuevos sujetos y actores" de los procesos migratorios. De igual manera, los de Falla (2005, 2007), Masís (2007), Narváez (2007), Ranum (2007), y aquellos estudios que involucran a diversos países de América Latina 
y del Caribe que, de manera acertada, combinan variables como la de la etnicidad y demografía, salud, cultura y educación. Específicamente el grupo de trabajo que realizó un estudio longitudinal de niños inmigrantes, conocido como CILS, en estos espacios (Jensen, 2001; Rumbaut y Portes, 2001). Acuña (2010), por su parte, ha puesto interés en las migraciones de niños, niñas y adolescentes vinculados con el trabajo infantil y derechos humanos en la región centroamericana. Los identifica como los nuevos actores en la migración. También el Programa Internacional para la Erradicación del Trabajo Infantil (IPEC, 2014) ha observado las migraciones de niñas, niños y adolescentes trabajadores intrarregionales en zonas transfronterizas en Centroamérica y República Dominicana que viven similares situaciones a las de los guatemaltecos que se desplazan por motivos laborales hacia la frontera sur de México.

Por otro lado, y de forma oportuna, durante los últimos años han surgido informes y diagnósticos de organismos internacionales y de la sociedad civil que llaman la atención sobre el preocupante estado en que se encuentra la niñez y adolescencia centroamericana y mexicana transitando sin compañía hacia Estados Unidos. Uno de ellos es el publicado por el Instituto Interamericano de Derechos Humanos (IIDH, 2016), mismo que aborda las "Prácticas relevantes de protección a los derechos de niños, niñas y adolescentes migrantes sin compañía en el Triángulo Norte y México"; también se encuentra el de la Comisión Nacional de Derechos Humanos (2016), que publicó el "Informe sobre la problemática de nińas, niños y adolescentes centroamericanos en contexto de migración internacional no acompańados en su tránsito por México y con necesidades de protección internacional". O el emitido por Voces Mesoamericanas, Acción con Pueblos Migrantes, A. C. (2016), informe que denominó La frontera me cruzó. De similar importancia es el reporte "Niñez y migración en Norte y Centro América: Causas, Políticas, Prácticas y Desafíos" (Musalo et al., 2015) realizado por un conjunto de académicos e integrantes de organismos no gubernamentales en un esfuerzo coordinado por la University of California Hastings College of the Law y la Universidad Nacional de Lanús, Argentina. Recientemente encontramos el trabajo coordinado por Kids In Need of Defense, KIND, y el Centro de Derechos Humanos Fray Matías de Córdova (2017), en la 
fronteriza ciudad de Tapachula, Chiapas. Este informe destaca las formas de violencia a las que se enfrenta la niñez migrante centroamericana así como la manera en que las experiencias de violencia sexual y por motivos de género determinan su decisión de emigrar.

Los estudios, reportes, informes y diagnósticos realizados durante estos últimos años sobre niñez y adolescencia viajando sin compañía de un adulto son un claro indicativo del contexto no favorable que presiona a la progresiva movilidad internacional, pero sobre todo las problemáticas y riesgos a que se enfrentan tanto en países de origen, de tránsito y destino. Alertan sobre los irregulares procesos de aprehensión y su posterior deportación que se realiza de manera poco segura e institucionalizada. El informe de KIND, por ejemplo, destaca que ningún país de la región ofrece, de manera integral, asistencia para que estos niños tengan una debida reintegración y garantía de seguridad básica para su reincorporación social y familiar una vez que son devueltos a sus países. Especialmente riesgoso es para aquellos que huyeron de la influencia y acoso de maras y de la violencia intrafamiliar vinculado con la ausencia de políticas de atención a personas menores de edad. Su retorno, a través de la deportación, no es favorable ni siempre recomendable, por eso muchos de ellos y ellas solicitan asilo y refugio, difícil y limitadamente otorgado por México y Estados Unidos.

\section{Niñas, niños y adolescentes detenidos y devueltos desde Tapachula, Chiapas ${ }^{3}$}

Entre 2012 y 2013 logré recoger algunas narrativas de un grupo de 15 niñas, niños y adolescentes de entre 11 y 13 años, provenientes de El Salvador y Honduras, entre ellos dos de Guatemala, en ese momento detenidos en el Albergue del DIF en Tapachula. Nueve eran niños, cinco nińas, más uno autorreconocido gay. La mayoría, trece, iniciaron

\footnotetext{
${ }^{3}$ Las niñas y niños que se encontraron en el albergue para el menor migrante del DIF en Tapachula, Chiapas, fueron "asegurados" por INM en distintas regiones de México, especialmente en la frontera sur. En los momentos del encuentro estaban a la espera de que el consulado de su país gestionara su devolución, según los convenios establecidos con el gobierno mexicano para tal fin. Debido al objetivo planteado en este artículo no se aborda en profundidad los temas de las estructuras y arreglos familiares en el país de origen. Eso sí, se tomaron partes resumidas de sus testimonios, mismos que se conjugaron con información de campo recopilada con otros actores involucrados, en Tapachula, Chiapas, México.
} 
su travesía migratoria para la reunificación familiar, los otros dos por la violencia intrafamiliar y de pandillas; especialmente quien se dijo gay salió huyendo de casa porque su padre no aceptó su condición y preferencia sexual, por lo que frecuentemente era discriminado y golpeado. Casi todos provenían de familias de alta tradición migratoria que han alimentado la idea de migrar "al norte". Desde la primera infancia han escuchado y socializado, de manera cotidiana entre sus parientes y amigos, la "necesidad" de viajar "al norte" para el reencuentro con uno de sus progenitores. Particularmente de aquellos cuyas madres emigraron cuando eran pequeños. Fueron encargados al cuidado de parientes cercanos. En los testimonios recurren al padrastro, la madrastra, medios hermanos y sobre todo a las abuelas — maternas, la mayoría — como las principales personas con quienes se relacionan durante la ausencia del progenitor o progenitora. La mitad de este reducido universo reveló que su madre migró hacia Estados Unidos cuando ellos tenían entre tres y cuatro ańos; y ahora, ya con 11 o 12 ańos, emprendieron la travesía con el anhelo del reencuentro. Esa misma proporción es la de mujeres que procrearon a sus hijos cuando ellas eran adolescentes, y que han vivido con distintas parejas con las cuales han concebido más hijos.

En las conversaciones, una parte del grupo destacó el tema de su familia, no tanto lo que percibía emocionalmente en ese momento en el albergue. Se referían a que, durante la primera etapa, la madre que había emigrado realizaba llamadas telefónicas transnacionales de manera periódica, en tanto encontraba un trabajo relativamente estable, pero al paso de los años la comunicación, de ella con sus hijos o las personas responsables de su cuidado, se producía en tramos más prolongados entre una llamada y otra. El envío de mensajes y fotos y el uso de la Internet median los vínculos familiares y favorecen la construcción ideal de que el hijo/hija un día será mandado a traer por su progenitora. En los casos narrados, tres niños y una niña señalaron que han transcurrido entre cinco y nueve años durante los cuales no ha sido posible el anhelado encuentro. En tanto, en el norte, eventualmente la madre ha encontrado otra pareja con la cual ha procreado otros hijos; "mis 'nuevos' hermanitos del norte”, señalaron dos de ellos. En la comunicación madre-padre-hijos, sobre todo con los hijos adolescentes, se alimenta 
invariablemente el deseo de ambas partes de la reunificación familiar, por lo que la expectativa del futuro de estos hijos no se vislumbra en su país de origen sino en el otro, con la madre. La idealización de una vida en el norte es muy compleja, tiene una expectación positiva y altamente valorada, aunque la realidad muchas veces muestra desencuentros entre personas que prácticamente son desconocidas cuya relación ha sido virtual. Pero este tema no se aborda en el presente artículo.

Ocurren ciertos acontecimientos que impulsan la decisión de la madre, en Estados Unidos, a agilizar el tan esperado viaje del hijo o hija que reside en Centroamérica. La queja de un conflicto entre primos y/o entre hermanos, tutelados por la abuela, favorecida en gran medida por los cambios en la adolescencia - coquetear con las chicas o chicos, el inicio de la vida sexual, necesidad de comprar un celular, ropa de moda, música moderna, el acceso a la Internet- puede ser el detonante o el acto que determina la decisión de migrar. Otras causas se relacionan con la conclusión de la educación primaria o, en casos extremos, cuando se vuelven presa de las pandillas y maras para acrecentar su dominio; o aquellos que cayeron en el consumo de drogas. Realidades y afectaciones importantes que agilizan la disposición de la madre/padre por ubicar los procedimientos necesarios para preparar el viaje de su hijo/hija. Si la madre no tuvo las posibilidades económicas para enviar por él o ella, ésta con ayuda de parientes o amigos consiguió el dinero para emprender la travesía. Dependiendo de la cantidad de dinero que reunió y de los contactos establecidos, los tutores o la madre, en Estados Unidos, logran organizar el viaje.

Por tratarse de un procedimiento irregular, en tanto que la madre también tiene una estancia indocumentada en Estados Unidos, se recurre a la contratación de los servicios del traficante de personas - pollero- en el mismo lugar de su residencia, o bien en el país de origen donde el tutor se hace cargo de contratar sus servicios para enviar a los hijos a Estados Unidos. No hay un solo patrón que indique los procedimientos en que la madre/padre, en el exterior, organice de manera conjunta con los parientes tutores el plan de viaje hacia el norte. Varios de los niños y niñas en custodia del Albergue en Tapachula fueron enviados desde sus países con traficantes polleros que los abandonaron cuando 
fueron aprehendidos por agentes de INM. Debido a esa particularidad se les clasificaron como migrantes sin compañía, o viajando solos.

Una vez que ingresaron a México y fueron detenidos o "asegurados" por las autoridades migratorias, inicia el periplo que concluirá generalmente con la deportación y limitadamente con el otorgamiento de asilo o refugio, como lo narran los autores del documento testimonial “¿Protección o captura de la infancia? Menos muros y más herramientas para el Sistema de protección integral a la infancia”, en este mismo número de EntreDiversidades. Quienes tenían menos de doce años fueron trasladados al alberge temporal del DIF; las niñas mayores de 12 y hasta 17 años fueron ubicadas en un lugar anexo al Albergue y a los hombres adolescentes los enviaron a la Estación Migratoria Siglo XXI para su posterior repatriación.

Ha sido tal el aumento de ingreso y deportación de las y los migrantes centroamericanos, incluidos menores de edad, durante los últimos años que las instancias migratorias han fortalecido el control y detención sobre todo en el sureste mexicano, particularmente en aquellos puntos que componen los dos cinturones de control ideados desde el Plan Sur: Chiapas-Tabasco, Oaxaca-Veracruz-Tabasco. ${ }^{4}$ Situación que se incrementó a partir del Programa Frontera Sur que se echó a andar justo en 2014, cuando la Casa Blanca declaró una "situación humanitaria urgente” al reconocer la detención de 56 mil niñas, niños y adolescentes migrantes irregulares procedentes de Guatemala, Honduras, El Salvador y México.

\footnotetext{
${ }^{4}$ En el año 2001, durante la administración del presidente Fox, se puso en marcha el Programa Plan Sur, mismo que establecía dos cinturones de control migratorio para fortalecer la estrategia de contención del flujo centroamericano de migrantes en tránsito por México. El Plan se basó en cinco acciones: fortalecer las actividades de inspección y control de los migrantes en las zonas del Istmo, Golfo de México o Pacífico; sumar esfuerzos institucionales para combatir el tráfico de indocumentados; obtener el máximo provecho posible de los recursos disponibles de las delegaciones regionales en materia de inspección y control; incrementar el número de deportados — asegurados - y detenciones de traficantes de indocumentados; y sumar a este esfuerzo interinstitucional el apoyo de los gobiernos de los estados y municipios (Casillas, 2007: 20). Este programa antecede al actual Programa Integral de la Frontera Sur, inaugurado en junio de 2014 durante el gobierno de Peña Nieto.
} 


\section{Detención y deportación. Ausencia de políticas integrales y los debidos procesos} Explicar esta compleja problemática requiere enfoques múltiples. En este artículo sólo se retoman algunos aspectos analizados por estudiosos que la han observado de manera sistemática, así como la observación e información propia surgida del ejercicio etnográfico en los años 2012, 2013 e incluso 2014. Por principio, se parte de la certeza de que no es problema que corresponda atender exclusivamente a un país, sino al conjunto de países, por lo que es adecuado enfocar la situación desde un contexto regional (CNDH, 2016; Villafuerte y García, 2016; Lomelín, 2017). Uno de los puntos clave es identificar las políticas migratorias del corredor Centroamérica-México-Estados Unidos que, en gran medida, tienen como finalidad interrumpir o amilanar el flujo migratorio irregular del sur hacia el norte. En Estados Unidos, al igual que sus antecesores, el gobierno de Obama fue un gran impulsor de esta política, mediante distintas reformas. Durante la gestión de su gobierno se consumó el mayor número de deportaciones de centroamericanos y mexicanos. La alerta que significó para su gestión presidencial la llegada, en 2014, de los miles de niños y adolescentes que viajaron sin compañía a los estados del sur de Estados Unidos, empujó para acordar con los gobiernos de México y Centroamérica - Guatemala, Honduras y El Salvador- medidas concretas con las que presionar, aún más, la migración irregular, especialmente de menores, por tratarse de un tema sensible que implica recursos económicos y jurídicos, ya que en Estados Unidos la ley prohíbe hacer deportaciones de menores de edad sin que medie un juicio legal.

En esa coyuntura el gobierno mexicano intensificó su habitual política de contención migratoria en su frontera sur al crear y ejecutar, en julio de 2014, el Programa Integral de la Frontera Sur — le antecedió el Plan Sur-, cuyo objetivo es la detención y deportación de migrantes 
irregulares centroamericanos que transitan por su territorio. ${ }^{5}$ El Programa cuenta con recursos de la Iniciativa Mérida suministrados por el gobierno de Estados Unidos (Villafuerte y García, 2016). En dos años de ejercicio, de mediados de 2014 hacia el primer semestre de 2016, aumentaron las detenciones y deportaciones de migrantes centroamericanos: en 2014 fueron detenidos — presentados- 118,446 migrantes del Triángulo Norte, y los deportados — devueltos- sumaron 104,269; en 2015, los detenidos alcanzaron la cifra de 170,323, siendo deportados 150,170. El comparativo entre 2014 y 2015 proyectó un aumento de 43.7 y $44.02 \%$, respectivamente. La efectividad del Programa Frontera Sur se demostró al disminuir las deportaciones de Estados Unidos, éstas fueron compensadas por el aumento de las deportaciones efectuadas por el gobierno mexicano. El mayor número de personas deportadas provenía de Guatemala, 70,493; seguido de Honduras, 50,527, y El Salvador, 29,154 (CNDH, 2016; Villafuerte y

\footnotetext{
${ }^{5}$ Esto se entiende en el marco del programa de Repatriación Ordenada y Segura, Digna y Humana del INM que fue la columna vertebral de las acciones de repatriación y columna básica de la política migratoria en México en su vínculo con los países vecinos: Estados Unidos y Centroamérica (Fernández de Castro, 2006). Antecedentes de ese Programa se encuentran desde los años ochenta cuando el Estado mexicano estableció una agenda migratoria con Guatemala en la que se idearon mecanismos bilaterales y multilaterales de repatriación ordenada y segura. De esa necesidad surgió la propuesta de una Política Migratoria Integral para la Frontera Sur en referencia a las relaciones con los países del Istmo centroamericano. El primer programa para establecer mecanismos de regulación migratoria se concretó en 1989 a través del Grupo binacional México-Guatemala sobre Asuntos Migratorios. En 1992 se acordó que serían objeto de repatriación por la frontera México-Guatemala los indocumentados de origen centroamericano. En relación con la repatriación de menores de edad se estableció, en 1993, que fueran llevados ante la representación consular guatemalteca para su documentación, previa a la deportación, y que el retorno se efectuara de forma separada de los adultos. En 2002 se suscribió el Primer Arreglo para la Repatriación Segura y Reordenada de Extranjeros Centroamericanos en las Fronteras de México y Guatemala con el objeto de facilitar la repatriación de indocumentados de Guatemala, El Salvador, Honduras y Nicaragua hasta su lugar de origen, pero en realidad los centroamericanos eran dejados en Guatemala, lo que creó problemas a aquel país por no poder trasladar a los otros hasta sus lugares de origen. Fue hasta 2005 que el procedimiento y los arreglos se ampliaron y el gobierno de Guatemala se comprometió a hacer los convenios para que los gobiernos de El Salvador y Honduras recibieran a sus nacionales; así es que los nacionales salvadoreños eran entregados por las autoridades de Guatemala en la frontera de Pedro de Alvarado, Guatemala/La Hachadura, El Salvador; asimismo los nacionales hondureños son entregados por las autoridades guatemaltecas en la frontera de Agua Caliente, Guatemala/Agua Caliente, Honduras. Hoy día han avanzado en el procedimiento de entrega de los menores de edad en cada país, sin lograr un programa integral de reintegración.
} 
García, 2016). Es decir, las políticas de ambos gobiernos han apostado por el reforzamiento de medidas que llevan a la detención y deportación de la migración irregular, más que promover e impulsar otro tipo de proyectos integrales regionales que desestimulen la emigración.

¿Cuáles son los efectos y el impacto que esta situación genera entre los niños y adolescentes no acompañados que transitan por el corredor que inicia en el norte de Centroamérica, que atraviesa México y concluye en sur de Estados Unidos? Vimos en el apartado anterior que los niños y nińas fueron detenidos en cualquier punto del país y trasladados al albergue y a la Estación migratoria Siglo XXI en Tapachula para su deportación. Sin embargo, estos y otros niños experimentan lo reportado por la Red Regional de Organizaciones Civiles para las Migraciones (RROCM, 2014) que, al igual que Musalo y sus colaboradores (2015), sintetizan para explicar la situación: en primera instancia ubican los peligros y la vulnerabilidad que se potencializa cuando recorren los países y atraviesan sus fronteras. Los principales riesgos son: sufrir lesiones físicas; ser cooptados por las redes del crimen organizado; ser sometidos a explotación sexual o laboral; sobrellevar maltrato psicosocial por parte de las instituciones en el momento de la repatriación, y aun pueden llegar a perder la vida. Asimismo pueden padecer enfermedades, sobre todo gastrointestinales por las irregularidades y calidad en el consumo de alimentos; heridas en los pies cuando las caminatas se prolongan; picaduras de animales e insolación. Durante la época de lluvia sobresalen las infecciones respiratorias asociadas a las fiebres. La sensación de permanente inseguridad e imprecisión en las jornadas de viaje generan constantes pesadillas, insomnios expresados en náuseas, vómitos, dolores de cabeza y estómago, provocados en gran medida por la ansiedad.

También se pueden encontrar con el abuso de poder, y en ocasiones el maltrato y extorsión de parte de agentes policiacos de distintos niveles: municipal, estatal, federal, Marina, Fronteriza, ejército, así como de los agentes de INM, es constante. O de bandas organizadas o grupos que les pueden ocasionar asaltos, robos, violaciones físicas y, a veces, como se dijo, muerte. 
La detención —aseguramiento-y devolución en y desde México

Cuando se da el momento de la detención — “aseguramiento", llamado así por INM-, normalmente son abandonados o separados de los guías o polleros, es decir, son trasladados al albergue del DIF. Esa situación multiplica la angustia porque son apartados del único vínculo conocido que tienen desde el lugar de origen. Para ellos, el traficante - pollero- es una persona clave que conoce los nexos con los parientes en el lugar de origen y también quien tiene el contacto con la madre/padre en el norte, en el lugar de destino. La sensación de soledad e incomunicación, al ser abandonado/separado de ese personaje, provoca tensión e incertidumbre por no saber cuál será su futuro inmediato. Estas emociones me fueron expresadas en las narrativas obtenidas en el albergue del DIF en Tapachula. Debido a la ausencia de información y de algún actor que gestione directamente sus derechos en esa condición, sienten impotencia y miedo; hay una violación a sus derechos y ausencia del debido proceso. Al final, después de varios días, con las gestiones por parte de los cónsules, y apoyo del INM, se da la deportación; sólo en circunstancias excepcionales se inicia un proceso de solicitud de asilo o refugio. En ocasiones, cuando algunos de éstos logran escapar al control migratorio, quedan en "condición de calle", esto los hace susceptibles a la explotación laboral, sexual, acoso y trata, favorecido por las deudas que adquieren. Asimismo, el pedir comida y dinero en la calle los vuelve muy endebles y sujetos fáciles para escenarios de alcoholismo y drogadicción. Situaciones como éstas se acentúan entre quienes permanecen más tiempo, por espacios más o menos prolongados, fuera del cuidado de tutores adultos o instituciones de servicio social —residencias o albergues temporales de asistencia pública o de la sociedad civil.

\section{La detención en Estados Unidos}

De la detención se hace cargo la Oficina de Aduanas y Protección Fronteriza, CBP. Musalo y otros (2015) apuntan que no existen condiciones adecuadas cuando son retenidos quienes emigran, lo que provoca la violación de los derechos elementales de los niños en virtud de las leyes federales y el derecho internacional de derechos humanos. 
CBP transfiere a los niños centroamericanos no acompañados a la Oficina de Reasentamiento de Refugiados, ORR, para su custodia a más largo plazo. Por su parte, niños y adolescentes mexicanos son repatriados de forma inmediata. Muchos de estos centros se encuentran distantes de los servicios legales, médicos y de salud mental, lo que impide el acceso de los detenidos a estos servicios. ORR ha acelerado significativamente el proceso de puesta en libertad de los niños y los adolescentes bajo su custodia con el fin de responder al aumento del número de los no acompañados que han llegado a Estados Unidos en los últimos años. En ocasiones, algunos son entregados a adultos que no son examinados por ORR, lo que los expone al abuso y a la inseguridad. Una vez en libertad, sólo un pequeño porcentaje de ellos recibe servicios de seguimiento para ayudarle a adaptarse a su nueva vida y familia en Estados Unidos, o para abordar cualquier trauma anterior durante la migración o en sus países de origen.

Otro efecto es la separación familiar, ya que las leyes de inmigración de Estados Unidos no dan prioridad a la reunificación familiar, y las alternativas para que familiares de personas que residen en Estados Unidos puedan inmigrar regularmente no siempre satisfacen las necesidades de beneficios. Una vez detenidos o deportados, los padres o tutores pierden el control de las decisiones relacionadas con la custodia y el cuidado de sus hijos, y tienen apuros o dificultades para poder comunicarse con ellos.

Por otro lado, Musalo y sus colaboradores (ídem) detectaron deficiencias procesales, ya que ese país no ha adoptado el criterio del interés superior del niño migrante; con lo cual los procedimientos existentes no se basan en ninguna evaluación que tenga en cuenta este criterio. Puede expulsarlos sin el derecho a un abogado designado, lo que vulnera las garantías procesales. La gran mayoría de los niños no acompañados no cuenta con un defensor de su causa - o tutor-, al igual que en México, que les asesore o apoye en el proceso de inmigración, en contravención de las normas internacionales.

Otras dificultades se encuentran en el momento de la deportación y lo que debería ser un proceso de reintegración. Estados Unidos, como México, no se involucra en acciones que conlleven a asegurar su 
integridad en el momento de la devolución a pesar de que lo requieren sus leyes federales. Ambos países los devuelve sin identificar si niños y adolescentes corren peligros al ser entregados a las oficinas encargadas de recibirlos, debido a que muchos de ellos huyeron de la violencia intrafamiliar o de las maras.

\section{Consideraciones finales}

Las migraciones de las niñas, niños y adolescentes no son nuevas. La mayoría de las decisiones para emigrar se relaciona con las limitadas o cambiantes estructuras de oportunidades para los padres, los jóvenes y los niños; el desempleo, deprimidos mercados regionales y nacionales, y los desastres medioambientales, son también causas de expulsión. En los casos observados predominó la reunificación familiar como el principal motivo para su desplazamiento, o mejor dicho su frustrado intento de migrar hacia Estados Unidos. Durante los últimos ańos la violencia y delincuencia han jugado un papel importante en los detonantes de las decisiones familiares e individuales.

El propósito de este artículo ha sido mostrar sólo un fragmento de una de las amplias posibilidades de investigación en los análisis migratorios en relación con la niñez y adolescencia migrante. Es imperativo identificar las incertidumbres y contingencias que contextualizan parte del proceso migratorio caracterizado por el tránsito indocumentado y los riesgos y peligros que esa condición conlleva. El viaje y la trayectoria interrumpida que emprenden cuando inician su travesía hacia Estados Unidos se ve cargada de riesgos al exponerse a personas o cosas que actúan como potenciales amenazas y colocan a aquel en una situación riesgosa y la vulnerabilidad que identifican esa situación. La vulnerabilidad se sitúa, en estas circunstancias, como la "falta o carencia de poder", que se acentúa en personas menores de edad como sujetos de derechos humanos. De modo que se trata de una condición o situación impuesta al migrante por estructuras de poder en el país de tránsito y destino, estructuras que no corresponden únicamente al Estado, sino que trascienden ese ámbito y recorren todo el tejido de relaciones sociales. Separación familiar, deportación y asistencia para la reintegración son dificultades a las que se enfrentan, ya que no siempre son sujetos a los 
debidos procesos. Ningún país involucrado ofrece asistencia, de forma integral, para su reintegración y garantía de seguridad básica que facilite su reincorporación social y familiar.

$\mathrm{Al}$ igual que los autores y los informes referidos hay coincidencia en que es urgente dar un tratamiento regional e integral al problema en su conjunto. El fenómeno de los niños en el contexto de la migración en Centroamérica, México y Estados Unidos debe abordarse a través de respuestas regionales. Los acuerdos existentes, así como los programas relativos a la migración en la región, están lejos de constituir una respuesta adecuada. Los tratados vigentes carecen de un sistema vinculante basado en derechos y de mecanismos de control para garantizar su cumplimiento. De igual forma hay que considerar el hecho de que un número creciente de niños, niñas y adolescentes se ha asentado en la región del Soconusco, sureste de México, y no hay políticas públicas que den protección a sus necesidades, además de las dificultades que implica la obtención de permisos de residencia (Musalo y otros, 2015).

El problema presenta múltiples retos. Abordarlo en sus distintos niveles, desde el origen, tránsito, destino y retorno, es responsabilidad de los Estados en sus distintos ámbitos de gobierno. Se requiere cambiar los enfoques privativos que han permeado los abordajes de contención y expulsión, y posicionar el interés superior del niño, niña $\mathrm{y}$ adolescente en el primer plano.

\section{Bibliografía citada}

Acnur, OIT, OIM, Unicef, 2013, Observación General Conjunta CDNCTM sobre los derechos humanos de la niñez en el contexto de la migración internacional, Nota conceptual, [en línea] disponible en http://plataformadeinfancia.org/wp.../02/CRC_CMWGeneral-Comment-_7864_S-2.doc [fecha de consulta: 26 de noviembre de 2017].

Acuña, Guillermo, 2010, Migración deniñas, niñosy adolescentes. Derechos humanos y trabajo infantil. Los nuevos actores en la migración y su incorporación al mercado de trabajo en la región: algunos elementos 
para su análisis, Plataforma subregional sobre trabajo infantil y adolescente: Guatemala, Honduras, El Salvador, Nicaragua, Costa Rica, Panamá y República Dominicana, DNI, Costa Rica. Arriola, Aura M., 1995, Tapachula, "la perla del Soconusco", ciudad estratégica para la redefinición de las fronteras, Flacso, Guatemala. Blanco, Blanca, 2014, “Migración femenina, 'trabajo muerto' y nichos sociolaborales: empleadas domésticas guatemaltecas en Tapachula, Chiapas, en Carolina Rivera Farfán (coordinadora), Trabajo y vida cotidiana de centroamericanos en la frontera suroccidental de México, CIESAS, México, pp. 107-136.

Caballeros, Álvaro, 2006, Derribando muros. La realidad de la niñez y adolescencia migrante en la frontera Guatemala-México, Defensoría de la Población Desarraigada y Migrante, Defensoría de la Niñez y la Juventud, Unicef, Guatemala.

Casillas, Rodolfo, 2007, Una vida discreta, fugaz y anónima. Los centroamericanos transmigrantes en México, CNDH, IOM, México.

Comisión Nacional de Derechos Humanos, CNDH, 2016, Informe sobre la problemática de niñas, niños y adolescentes centroamericanos en contexto de migración internacional no acompañados en su tránsito por México, y con necesidades de protección internacional, [en línea] disponible en http://www.cndh.org.mx/sites/all/doc/ Informes/Especiales/Informe_NNACMNA... [fecha de consulta: 15 de julio de 2017].

Conapo, Fundación Bancomer, BBVA Research, 2016, “Anuario de Migración y Remesas”, México, diciembre, 2015, [en línea] disponible en http://www.migracionyremesas.org/ $\mathrm{tb} /$ ?a=F12358 [fecha de consulta: 21 de julio de 2017].

Consejo Nacional de Evaluación de la Política de Desarrollo Social, Coneval, 2015, Coneval informa los resultados de la medición de pobreza 2014, Dirección de Información y Comunicación Social, [en línea] disponible en https://www.coneval.org.mx/ SalaPrensa/Documents/Comunicado005_Medicion_pobreza_2014.pdf [fecha de consulta: 20 de mayo de 2017].

Corona, Rodolfo, 2008, "Metodologías cuantitativas y fuentes de información para el estudio de las migraciones”, Diplomado en 
Estudios Migratorios en la Frontera Sur de México, El Colegio de la Frontera Sur, San Cristóbal de Las Casas, Chiapas.

Cranshaw, Martha y Abelardo Morales, 1998, Mujeres adolescentes y migración entre Nicaragua y Costa Rica, Flacso, Unión Europea, Programa Mujeres Adolescentes y Consejo de Integración Social, Costa Rica.

Escobar Sarti, Carolina, 2008, Los pequeños pasos de un camino minado: Migración, niñez y juventud en Centroamérica y el sur de México, Consejería de Proyectos, Guatemala.

Falla, Ricardo, 2005, Alicia. Explorando la identidad de una joven maya. Ixcan, Guatemala, Avancso y Editorial Universitaria, Guatemala. Falla, Ricardo, 2007, Migración transnacional retornada. Juventud indigena de Zacualpa, Guatemala, Avancso y Editorial Universitaria de la Universidad de San Carlos Guatemala, Guatemala.

Fernández de Castro, Rafael, 2006, Migración y repatriaciones. México en la encrucijada Norte Sur, Rosa Ma. Porrúa Ediciones, México. Instituto Interamericano de Derechos Humanos, IIDH, 2016, Prácticas relevantes de protección a los derechos de niños, niñas $y$ adolescentes migrantes sin compañia en el Triángulo Norte $y$ México, San José Costa Rica, [en línea] disponible en http:// www.iidh.ed.cr/iidh/media/4829/migrantes-tn-web.pdf [fecha de consulta: 21 de julio 2017].

Jensen, Leif, 2001, “The Demographic Diversity of Immigrants and their Children”, in Rubén G. Rumbaut and Alejandro Portes, Ethnicities. Children of Immigrants in America, Berkely and Los Angeles California, University of California Press, Ltd., pp. 21-56.

KIND y Centro de Derechos Humanos Fray Matías de Córdova, 2017, Niñez interrumpida: violencia sexual y por motivos de género contra niñez migrante y refugiada centroamericana, [en línea] disponible en https://supportkind.org/wp.../06/NinezInterrumpida-En-español_Full-Version.pdf [fecha de consulta: 14 de agosto de 2017].

Lomelín, Julián, 2017, "Niñez en contexto de movilidad: Motivos, riesgos y Peligros", UIA, México, [en línea] disponible en http:// 
ibero.mx/prensa/ninez-en-contexto-de-movilidad-motivosriesgos-y-peligros [fecha de consulta: 18 de julio de 2017].

Masís, Karen, 2007, "En la escuela...también: niñas, niños y adolescentes inmigrantes nicaragüenses en el sistema educativo público de Costa Rica", en ECA Estudios Centroamericanos, "Migraciones: espacios transnacionales, nuevas dinámicas de poder e identidad", vol. 62, núm. 699-700, pp. 150-156, Universidad Centroamericana José Simeón Cañas, El Salvador.

Médicos Sin Fronteras, 2017, Forzados a huir del Triángulo Norte de Centroamérica: Una crisis humanitaria olvidada, [en línea] disponible en http://www.msf.mx/document/forzados-a-huirdel-triangulo-norte-de-centroamerica-una-crisis-humanitariaolvidada [fecha de consulta: 30 de junio de 2017].

Musalo, Karen, Lisa Frydman y Pablo Ceriani, 2015, Niñez y migración en Norte y Centro América: Causas, Politicas, Prácticas y Desafios, University of California Hastings College of the Law y Argentina, Universidad Nacional de Lanús, USA.

Narváez, Juan Carlos, 2007, Ruta transnacional: a San Salvador por Los Ángeles. Espacios de interacción juvenil un contexto migratorio, Universidad Autónoma de Zacatecas, Porrúa, Instituto Mexicano de la Juventud, México.

Organización Internacional para las Migraciones, OIM, 2014, Combate a la Trata de Personas, Género y Niñez Migrante. en http://oim. org. $\mathrm{mx} /$ ?portfolio=combate-a-la-trata-de-personas-genero-yninez-migrante [fecha de consulta:18 de septiembre de 2018].

Pavez, Iskra, 2012, “¿Quién decide la migración infantil?”, [en línea] disponible en https://gsia.blogspot.mx/2012/07/quien-decide-lamigracion-infantil.html [fecha de consulta: 1 de junio de 2017]. Pedone, Claudia, 2010, "Introducción. Más allá de los estereotipos: desafíos en torno de las familias migrantes", Grupo Interdisciplinario de Investigador@s migrantes (coordinador), Familias, niños, niñas y jóvenes migrantes. Rompiendo estereotipos, IEPALA, Madrid, pp. 11-16.

Programa Internacional para la Erradicación del Trabajo Infantil, IPEC, 2014, Niños, niñas y adolescentes migrantes trabajadores en zonas 
fronterizas en Centroamérica y Panamá, Oficina Regional para América Latina y el Caribe, Costa Rica. Ranum, Elin Cecile, 2007, "Pandillas juveniles transnacionales en CA, México y Estados Unidos”, Diagnóstico Nacional Guatemala, IUDOP, Universidad Centroamericana, San Salvador.

Red Internacional de Migración y Desarrollo, 2004, Publicaciones, vol. 2, [en línea] disponible en http://rimd.reduaz.mx/paginaContenidoBasico.php?id=42 [fecha de consulta: 9 de febrero de 2017].

Red Regional de Organizaciones Civiles para las Migraciones, RROCM, 2014, Declaración de la RROCM ante la XIX Conferencia Regional sobre Migración CRM. Managua, Nicaragua, junio de 2014, [en línea] disponible en http://www.crmsv.org/.../ RROCM\%20Declaración\%20ante\%20CRM\%20junio\%20 2014.pdf [fecha de consulta: 15 de agosto de 2017.

Rivera, Carolina, 2014, "Niños, niñas y adolescentes centroamericanos en el mercado laboral de la frontera Guatemala-México. Hacia la evidencia de una presencia encubierta y simulada”, en Carolina Rivera Farfán (coordinadora), Trabajo, empleo y vida cotidiana de centroamericanos en la frontera suroccidental de México, CIESAS, México, pp. 73-105.

Rojas, Martha, 2002, "Mujeres migrantes en la Frontera Sur de México", Foro Nacional Migración y Desarrollo, Memoria LVIII Legislatura de la Cámara de Diputados del Honorable Congreso de la Unión, Tapachula, Chiapas, pp. 45-52.

Rojas, Martha, 2005, "La situación de las mujeres migrantes en la frontera sur", Seminario mujeres y migración en las Fronteras de México, 8 de marzo, s.l.

Rumbaut, Rubén G. and Alejandro Portes, 2001, "Introduction. Ethnogenesis: Coming of Age in Immigrant America", in Rumbaut, Rubén G. and Alejandro Portes, Ethnicities. Children of Immigrants in America, Berkely and Los Angeles California, University of California Press, Ltd., pp. 1-19.

Villafuerte, Daniel y Ma. del Carmen García, 2016, "De la crisis de los niños migrantes a la crisis de la migración cubana: intereses 
geopolíticos y expresiones de la crisis del sistema migratorio Centroamérica-México-Estados Unidos entre 2014 y 2015”, Boletín de Antropología, Universidad de Antioquia, Medellín, vol. 31, núm. 52, pp. 15-33, [en línea] disponible en http:// dx.doi.org/10.17533/udea.boan.v31n52a03 [fecha de consulta: 14 de agosto de 2017].

Voces Mesoamericanas, Acción con Pueblos Migrantes, A.C., 2016, La frontera me cruzó, CDH Fray Matías de Córdova, San Cristóbal de Las Casas, Chiapas. 